\title{
Surgical and neurologic outcomes after robotic thymectomy in 100 consecutive patients with myasthenia gravis
}

\author{
Giuseppe Marulli, MD, PhD, Marco Schiavon, MD, Egle Perissinotto, MD, Antonella Bugana, MD, \\ Francesco Di Chiara, MD, Alessandro Rebusso, MD, and Federico Rea, MD
}

\begin{abstract}
Objective: Thymectomy is a well-defined therapeutic option for patients with myasthenia gravis; however, controversies still exist about the surgical approach, indication, and timing for surgery. We reviewed our experience reporting surgical and neurologic results after robotic thymectomy in patients with myasthenia gravis.
\end{abstract}

\begin{abstract}
Methods: Between 2002 and 2010, 100 patients (74 female and 26 male; median age, 37 years) underwent left-sided robotic thymectomy using the da Vinci robotic system (Intuitive Surgical, Inc, Sunnyvale, Calif). The Myasthenia Gravis Foundation of America classification was adopted for pre- and postoperative evaluation. Preoperative Myasthenia Gravis Foundation of America class was I in $10 \%$ of patients, II in $35 \%$ of patients, III in $39 \%$ of patients, and IV in $16 \%$ of patients.
\end{abstract}

Results: Median operative time was 120 (60-300) minutes. No death or intraoperative complications occurred. Postoperative complications were observed in 6 patients $(6 \%)$ (bleeding requiring blood transfusions in 3 , chylothorax in 1, fever in 1, and myasthenic crisis in 1). Median hospital stay was 3 days (range, 2-14 days). Histologic analysis revealed 76 patients $(76 \%)$ with hyperplasia, 7 patients $(7 \%)$ with atrophy, 8 patients $(8 \%)$ with small thymomas, and 9 patients $(9 \%)$ with normal thymus; ectopic thymic tissue was found in 26 patients $(26 \%)$. Clinical follow-up showed a 5-year probability of complete stable remission and overall improvement of $28.5 \%$ and $87.5 \%$. Remission was significantly associated with preoperative I to II Myasthenia Gravis Foundation of America class $(P=.02)$. A significant improvement rate was found in Myasthenia Gravis Foundation of America class I to II $(P=.03)$ and AbAchR $+(P=.04)$. A high percentage of patients interrupted or reduced their medications.

Conclusions: Robotic thymectomy is a safe and effective procedure. We observed a neurologic benefit in a great number of patients. A better clinical outcome was obtained in patients with early Myasthenia Gravis Foundation of America class. (J Thorac Cardiovasc Surg 2013;145:730-6)

Myasthenia gravis (MG) is a rare autoimmune disease that affects neuromuscular transmission. A causal role has been advocated for the thymus gland in the complex pathogenesis of this disease, and thymectomy is now an essential part of the integrated treatment of nonthymomatous MG. However, much controversy still exists regarding the selection of patients, extent of thymic resection, and appropriate surgical approach. ${ }^{1}$

A variety of surgical approaches for thymectomy have been described, ranging from open (basic transsternal ${ }^{2}$ or the more aggressive extended transcervical and transsternal maximal thymectomy ${ }^{3}$ ) to minimally invasive

From the Division of Thoracic Surgery, Department of Cardiologic, Thoracic, and Vascular Sciences, University of Padua, Padua, Italy.

Disclosures: Authors have nothing to disclose with regard to commercial support.

Read at the 38th Annual Meeting of The Western Thoracic Surgical Association, Maui, Hawaii, June 27-30, 2012.

Received for publication June 25, 2012; revisions received Sept 24, 2012; accepted for publication Dec 10, 2012; available ahead of print Jan 14, 2013.

Address for reprints: Giuseppe Marulli, MD, PhD, Division of Thoracic Surgery, Department of Cardiologic, Thoracic, and Vascular Sciences, University of Padua, Via Giustiniani, 2-35128 Padua, Italy (E-mail: giuseppe.marulli@unipd.it).

$0022-5223 / \$ 36.00$

Copyright (c) 2013 by The American Association for Thoracic Surgery

http://dx.doi.org/10.1016/j.jtcvs.2012.12.031 (transcervical ${ }^{4}$ or video-assisted thoracoscopic thymectomy ${ }^{5}$ ) approaches. Each approach has benefits and drawbacks. Only nonrandomized retrospective case series have been available for comparing operative approaches, and the heterogeneity of patient selection, different timing and type of surgery, several clinical classifications used, and different methods applied for evaluating the results, along with many other confounding factors, have made a comparative analysis complicated, if not impossible. ${ }^{1}$ Thus, a consensus on the best practice has not been reached.

Because of the lack of data from prospective thymectomy trials for patients with MG, clinicians must consider the best balance among the extent of resection, morbidity, patient acceptance, and results. The intrinsic characteristics of MG, the problems related to immunosuppression, and the generally young age of patients make the intraoperative and postoperative management of patients with MG challenging. In an attempt to find the best compromise between a complete thymic resection and minimizing the effect of surgery on the patient, video-assisted thoracoscopic thymectomy has been proposed as a valid alternative to open approaches for patients with nonthymomatous MG. The introduction of robotic instrumentation in the last decade has increased the potential technical skill and safety of standard 


\section{Abbreviations and Acronyms \\ CSR = complete stable remission \\ $\mathrm{IQR}=$ interquartile range \\ MG = myasthenia gravis \\ MGFA $=$ Myasthenia Gravis Foundation of America}

thoracoscopy and will eventually improve clinical results. ${ }^{6}$ However, almost all reported series on robotic thymectomy ${ }^{7-11}$ have involved relatively few patients and short follow-up periods. Furthermore, comparisons are difficult because of a lack of standardized nomenclature.

We report our experience with robotic thymectomy in 100 consecutive patients with MG and provide data on the surgical results and long-term neurologic outcomes, following the Myasthenia Gravis Foundation of America (MGFA) recommendations for MG clinical research standards. ${ }^{12}$

\section{PATIENTS AND METHODS}

A retrospective analysis of a prospectively maintained database was conducted for 100 patients affected by MG who received a unilateral, 3-port, left-sided robotic thymectomy (MGFA thymectomy classification: class T-2c) performed by the Thoracic Surgery Division in Padua, Italy, using the da Vinci robotic surgical system (Intuitive Surgical, Inc, Sunnyvale, Calif) between 2002 and 2010. All patients signed a detailed consent form describing possible complications of thymectomy via a robotic approach. The institutional review board approved this study.

The diagnosis of MG was based on clinical criteria, electromyography, edrophonium chloride test results (Tensilon; ICN Pharmaceuticals, Inc, Costa Mesa, Calif), and circulating AbAchR levels. Patients with negative AbAchR results also were evaluated for anti-MuSK antibodies and excluded from surgery when positive. Other exclusion criteria were radiologic evidence of invasive thymoma, the presence of adhesions (eg, previous thoracic surgery or pleuritis), the inability to perform single lung ventilation, or a body mass index greater than 35 . Twenty-six male patients $(26 \%)$ and 74 female patients $(74 \%)$ with a median age of 37 years (interquartile range [IQR], 25-47 years) were included in the study. The median duration of preoperative symptoms was 11 months (IQR, 5-24 months); 66 patients were AbAchR positive, and 34 patients were seronegative. Data on patient demographics, type and severity of MG, and intraoperative and postoperative events (eg, complications, need for open conversion or additional ports or accesses, operative time, and length of hospital stay) were collected.

Preoperative and postoperative clinical assessments were conducted by a team of neurologists. Preoperative evaluation included a neurologic assessment, chest radiography, computed tomography (or magnetic resonance imaging in selected patients), electrocardiography, and spirometry. Preoperative preparation included a reduction or an interruption of steroid treatment whenever possible. Plasma exchange therapy or intravenous immunoglobulin treatments were administered to patients at risk for postoperative respiratory failure (history of respiratory muscle involvement or recent worsening of symptoms) on the basis of the clinical judgment of a neurologist and previous effective use.

The MGFA recommendations for clinical research standards classification ${ }^{12}$ were followed for the clinical classification, preoperative and postoperative therapy status, post-intervention clinical status evaluation, and morbidity/mortality descriptions. Preoperative MGFA clinical classifications were as follows: class I in 10 patients, class IIa in 26 patients, class IIb in 9 patients, class IIIa in 24 patients, class IIIb in 15 patients, class
IVa in 8 patients, and class IVb in 8 patients. Preoperatively, 41 patients were receiving single-drug therapy (2-drug therapy in 39 and $\geq 3$ medications in 20). According to the MGFA recommendations, postintervention status was described as follows: complete stable remission (CSR) (patients without symptoms or signs of MG for at least 1 year and no therapy); pharmacologic remission (same criteria as CSR except that the patient continues to take some form of medication, excluding cholinesterase inhibitors); minimal manifestations (no symptoms or functional limitations but some weakness detectable by careful examination), changes in status (improved, unchanged, worse, exacerbation, and died of MG). CSR and pharmacologic remission were calculated at the end of a minimal 12-month follow-up period.

\section{Surgical Technique}

In all patients, an extended thymectomy with en bloc resection of the anterior mediastinal fat tissue was performed following Masaoka and colleagues' criteria. ${ }^{2}$ The adipose tissue around the upper poles of the thymus, both innominate veins, the retrosternal area, and the pericardium were resected meticulously. The resection borders were the diaphragm caudally, the thyroid gland cranially, and the phrenic nerves laterally. The surgical technique has been described. ${ }^{8}$ In our series, our preference was for a left-sided approach for technical, clinical, and anatomic reasons. As demonstrated by Rückert and colleagues, ${ }^{13}$ the larger part of the thymus is on the left side. Furthermore, in approximately $72 \%$ of cases $^{1}$ the thymus gland is located lateral and under the left phrenic nerve, which is better identified and safely dissected under direct vision from the left, whereas the right phrenic nerve is partially protected by the superior vena cava in the high mediastinum and may be easily identified and followed in the lower part. All mediastinal fat tissue was removed that comprised the aortopulmonary window, which is a frequent side of ectopic thymic tissue, and it is easier to reach by the left approach. To identify the location of the ectopic thymic tissue, the perithymic fat was sent for histopathologic examination, divided and classified on the basis of location: pretracheal infrathyroid fat, fatty tissue in the aortopulmonary window, mediastinal fat lateral to right and left phrenic nerves, and pericardial fat up to the diaphragm. Microscopically, the histologic types of the thymus were described as hyperplastic, involuted, and thymoma. The foci of ectopic thymic tissue with Hassall bodies in the excised cervicomediastinal fat were searched for with hematoxylin-eosin staining.

\section{Statistical Analysis}

Qualitative variables were summarized as frequency distributions. Quantitative data are described as median and first and third quartile (IQR). The normality of the data distribution was assessed by the Shapiro-Wilk test. The probabilities of CSR and improvement were calculated by cumulative analysis according to the Kaplan-Meier method. A univariate analysis to potentially predict CSR and improvement was conducted with the log-rank test. To fully understand the factors involved in the outcomes, all the variables were entered in multivariate analysis by applying the Cox regression model. Proportional hazards assumption was graphically checked. Differences in distributions were tested by means of the chi-square test or Fisher exact test, as opportune.

To evaluate the significance of change of drug dosage over follow-up, data on drug dosage were treated in the repeated-measures analysis with drug dosage as the dependent data and time as repeated measures effect (within-subject effect). Given that drug dosage was not normally distributed, nonparametric analysis of variance for repeated measurements was applied. All the statistical procedures were performed with SAS version 9.1.2 (SAS Institute, Inc, Cary, NC).

\section{RESULTS}

All procedures were carried out endoscopically, with no conversion to median sternotomy or thoracotomy. A fourth 
access was used in 3 patients: In 2 patients, a fourth access on the right side was used to introduce optics for better visualization of the right phrenic nerve, and in 1 patient, a cervicotomy was required to complete dissection of the thymic upper horns. No death or intraoperative complications occurred, and all patients were extubated in the operating room within 1 hour after surgery. The median operative time was 120 minutes (IQR, 106-159 minutes), median time to chest tube removal was 1 day (IQR, 1-2 days), and median hospitalization time was 3 days (IQR, 2-4 days). Postoperative complications occurred in 6 patients $(6 \%)$. In our early experience, 1 patient had a chylothorax on postoperative day 2 ; this prolonged hospitalization ( 2 weeks) and was successfully managed with conservative treatment. A hemothorax developed in 3 patients, but it did not require reoperation and was successfully managed with a chest tube and blood transfusions. Fever that required prolonged treatment with antibiotic therapy developed in $1 \mathrm{pa}-$ tient. A postoperative myasthenic crisis necessitated intubation and mechanical ventilation for 24 hours in 1 patient. Pathologic examinations revealed 76 thymic hyperplasias, 7 atrophies, 9 normal thymuses, and 8 small (1-3 $\mathrm{cm}$ ) thymomas. The median weight of the specimens (thymus and perithymic tissue) was $44 \mathrm{~g}$ (IQR, 31-56 g). The pathologic analysis of perithymic and mediastinal fat tissue identified the presence of ectopic thymic tissue in 26 patients $(26 \%)$. All patients were neurologically evaluated at 12 or more months after surgery (median, 67 months; IQR, 37-91 months). At the last follow-up (May 2012), 98 patients were alive, and 2 patients had died 25 and 29 months postoperatively of extrathymic cancers (gastric cancer and leukemia). No evidence of tumor recurrence was found in patients with thymoma. The 5-year CSR rate was $28.5 \%$, and the 5-year improvement (all patients with the following MGFA postintervention status: pharmacologic remission and minimal manifestation) rate was $87.5 \%$ (Figure 1). Median time to improvement was 17.9 months (IQR, 15-20.2 months). Univariate analysis of prognostic factors (Table 1) for CSR identified a significantly better result in patients with preoperative MGFA classes I and II compared with MGFA classes III and IV $(P=.002$; Figure $2, A$ ). A trend for a better CSR rate was seen in patients with an absence of ectopic thymic tissue $(P=.08$; Figure 2, B). A significant improvement rate was observed in patients with MGFA classes I and II $(P=.04$; Figure 2, $C)$ and positive AbAchR results $(P=.04$; Figure $2, D)$. No significant differences were observed in age, sex, histology, or symptom onset before surgery. The results from multivariate analysis confirmed the statistical significance of association between MGFA classes I and II and complete remission (multivariate adjusted $P=.02$ ) and between MGFA classes I and II and positive AbAchR and 5-year improvement (multivariate adjusted $P=.03$ and .04 , respectively). The post-intervention therapy status assessment

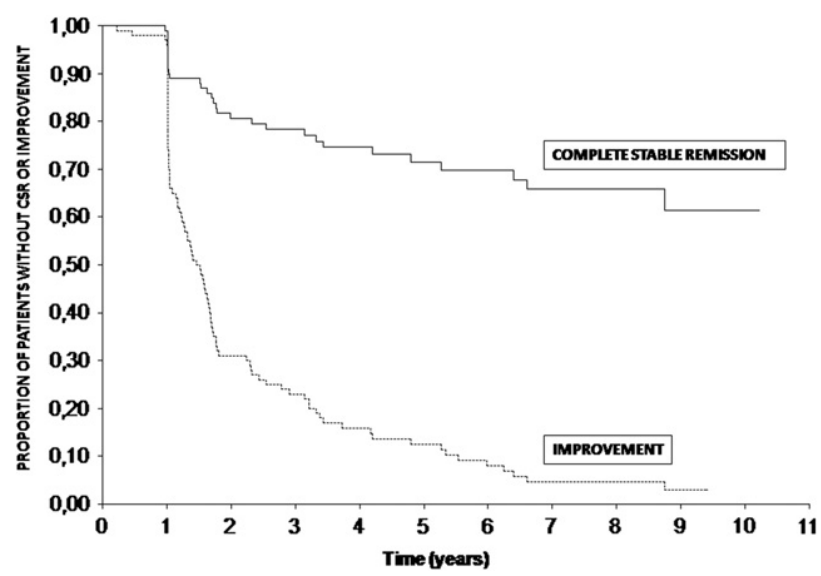

FIGURE 1. Cumulative probability of CSR and improvement. CSR, Complete stable remission.

(Table 2) showed a suspension or reduction in the median dose of cholinesterase inhibitor in $69.1 \%$ of patients and a suspension or reduction of prednisone in $89.4 \%$. Significant reductions in the median cholinesterase inhibitor dose $(P=.002)$ and median prednisone dose $(P=.0003)$ were observed over a 10-year follow-up compared with the preoperative dosage (Figure 3, $A$ and $B$ ).

TABLE 1. Statistical analysis of factors predicting complete stable remission and improvement

\begin{tabular}{|c|c|c|c|c|c|c|}
\hline Factor & $\begin{array}{l}\text { 5-y CSR } \\
\text { rate }(\%)\end{array}$ & $P^{*}$ & $\boldsymbol{P} \dagger$ & $\begin{array}{c}5-y \\
\text { improvement } \\
\text { rate }(\%) \\
\end{array}$ & $P^{*}$ & $\boldsymbol{P} \dagger$ \\
\hline Preoperative MGFA & & .002 & .02 & & .04 & .03 \\
\hline Class I-II $(\mathrm{n}=45)$ & 46.8 & & & 94.2 & & \\
\hline Class III-IV $(\mathrm{n}=55)$ & 18.2 & & & 84.8 & & \\
\hline Gender & & .86 & .77 & & .68 & .39 \\
\hline Male $(\mathrm{n}=26)$ & 30.5 & & & 86.4 & & \\
\hline Female $(\mathrm{n}=74)$ & 28.1 & & & 88.9 & & \\
\hline AchR antibodies & & .19 & .17 & & .04 & .04 \\
\hline Positive $(\mathrm{n}=66)$ & 23.4 & & & 91.3 & & \\
\hline Negative $(\mathrm{n}=34)$ & 40.7 & & & 83.1 & & \\
\hline Histology & & .32 & .41 & & .30 & .15 \\
\hline Benign thymus $(\mathrm{n}=92)$ & 31.3 & & & 86 & & \\
\hline Thymoma $(\mathrm{n}=8)$ & 12.5 & & & 100 & & \\
\hline Age & & .20 & .79 & & .31 & .39 \\
\hline$\leq 36.5$ y $(\mathrm{n}=50)$ & 39.4 & & & 91.6 & & \\
\hline$>36.5$ y $(\mathrm{n}=50)$ & 21.7 & & & 86 & & \\
\hline Ectopic thymic tissue & & .08 & .19 & & .49 & .63 \\
\hline Present $(n=26)$ & 21.4 & & & 95.2 & & \\
\hline Absent $(\mathrm{n}=74)$ & 33.2 & & & 86.7 & & \\
\hline $\begin{array}{l}\text { Preoperative duration of } \\
\text { symptoms }\end{array}$ & & .33 & .28 & & .84 & .91 \\
\hline$\leq 12 \mathrm{mo}(\mathrm{n}=58)$ & 32.8 & & & 91.4 & & \\
\hline$>12 \mathrm{mo}(\mathrm{n}=42)$ & 22.2 & & & 84.1 & & \\
\hline
\end{tabular}




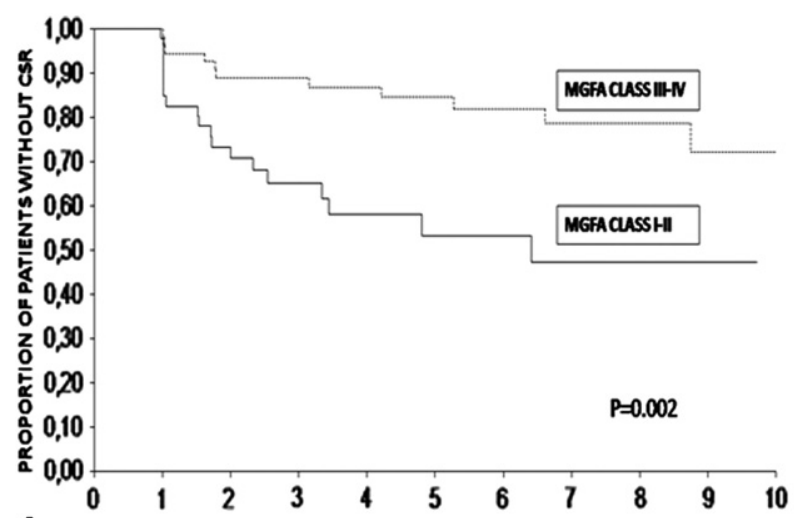

A
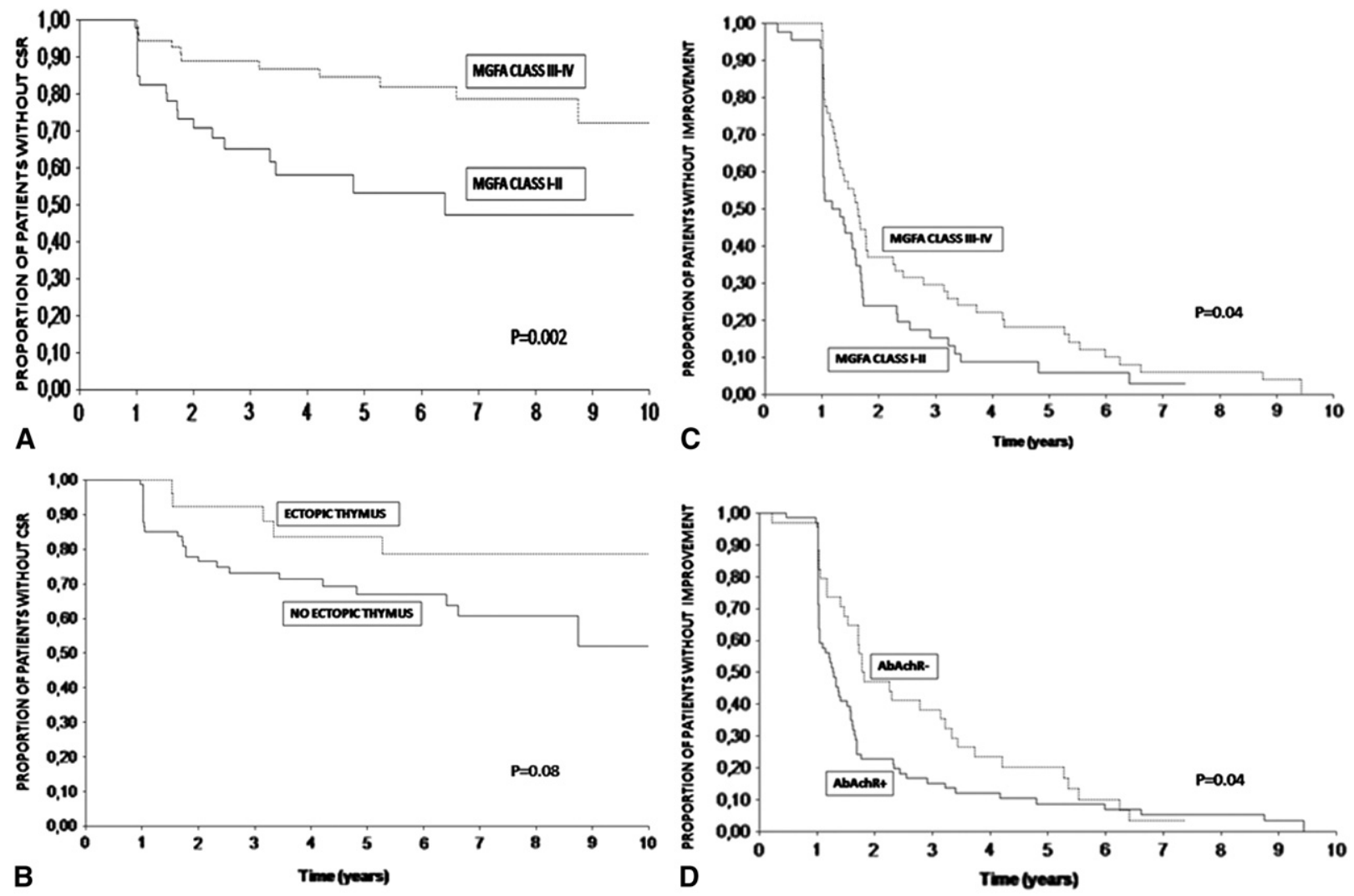

FIGURE 2. Cumulative probability of CSR on the basis of MGFA class (A) and presence or absence of ectopic thymus (B). Cumulative probability of improvement on the basis of MGFA class (C) and AbAchR status (D). CSR, Complete stable remission; MGFA, Myasthenia Gravis Foundation of America.

\section{DISCUSSION}

Despite general agreement on the beneficial role of thymectomy for patients with MG, no prospective randomized trial has examined whether thymectomy is superior to medical treatment, although an ongoing international multicenter randomized clinical trial is comparing thymectomy and therapy with prednisone alone. ${ }^{14}$ In addition to the low level of evidence supporting thymectomy, controversy also exists regarding the best surgical approach, the optimal extent of resection and degree of invasiveness, and the selection criteria for surgical candidates. Numerous thymectomy

TABLE 2. Myasthenia Gravis Foundation of America pre- and postintervention therapy status

\begin{tabular}{lcrr}
\hline & & \multicolumn{2}{c}{ Postoperative } \\
\cline { 3 - 4 } \multicolumn{1}{c}{ Drugs } & Preoperative & Interrupted & Reduced \\
\hline Cholinesterase inhibitors & $94(94 \%)$ & $36(38.3 \%)$ & $29(30.8 \%)$ \\
Prednisone & $56(56 \%)$ & $23(41.1 \%)$ & $27(48.2 \%)$ \\
Azathioprine & $26(26 \%)$ & $9(34.6 \%)$ & $5(19.2 \%)$ \\
Cyclosporine & $4(4 \%)$ & $4(100 \%)$ & \\
Plasma exchange therapy, & $11(11 \%)$ & $9(81.8 \%)$ & $2(18.2 \%)$ \\
$\quad$ chronic & & & \\
IVIg therapy, chronic & $12(12 \%)$ & $11(91.7 \%)$ & \\
\hline
\end{tabular}

IVIg, Intravenous immunoglobulin. techniques with different degrees of invasiveness have been described, all with similar results. ${ }^{2-11}$ Jaretzki $^{1}$ noted that these reports suffer from a lack of uniform reporting with respect to disease severity, extent of thymectomy, response to thymectomy, specimen histology, and follow-up length, as well as the application of nonstandard statistical methods. In 2000, the MGFA published recommendations for MG clinical research standards. A series of preoperative and postoperative classifications were proposed in an attempt to give a common and uniform language for further studies. ${ }^{12}$ Unfortunately, only a few studies have adopted these recommendations; thus, controversy, particularly regarding the best surgical approach, remains. Ideally, the less-invasive surgical technique is desirable, assuming the results are equivalent. Compared with conventional transsternal techniques, minimally invasive techniques have become increasingly popular because of their low procedural morbidity and mortality, short hospital stay, optimal cosmesis, minor surgical access trauma and postoperative pain, better preservation of pulmonary function, and similar efficacy. ${ }^{4,5,7,8,15}$ Thoracoscopic thymectomy combines the advantages of minimally invasive techniques with a good view of the anterior mediastinum, so that an extended thymectomy can be performed as needed. Disadvantages 

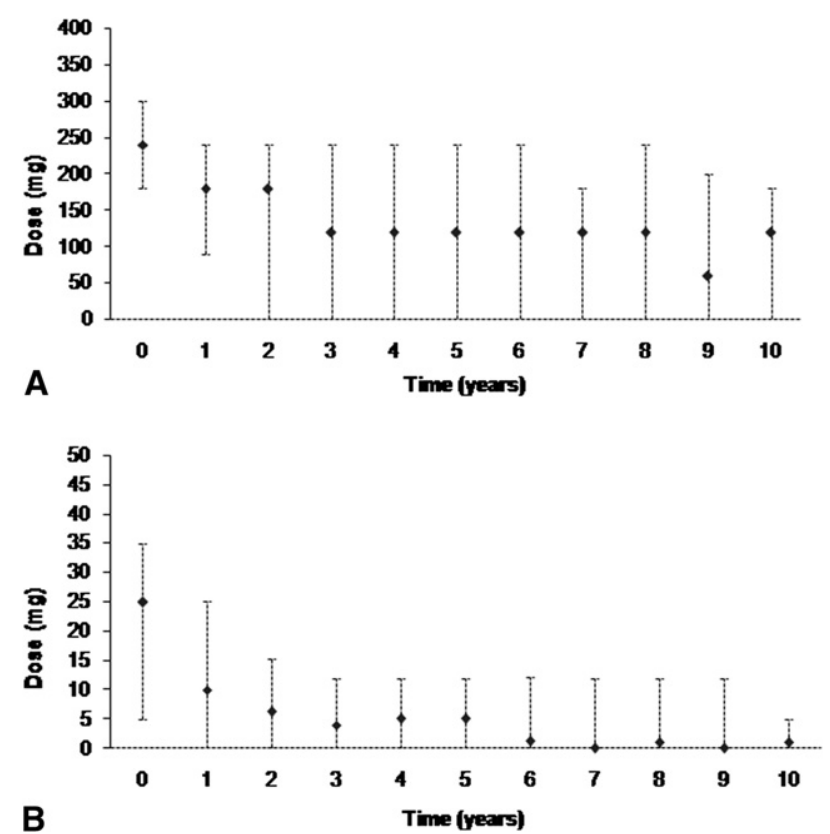

FIGURE 3. Postoperative reduction over time of the median dose of cholinesterase inhibitor (A) and prednisone (B) compared with the basal preoperative treatment Symbols are median, line at the lower end is the first quartile, line at the upper end is the third quartile.

of the conventional thoracoscopic technique include a 2-dimensional view of the operative field and the fact that the arms do not articulate, making it difficult to operate in the contralateral mediastinum and in the neck around corners in a fixed 3-dimensional space. In the last decade, robotic systems have been successfully introduced to thoracic surgery for treating various diseases, especially diseases of the anterior mediastinum. ${ }^{16}$ The da Vinci surgical system consists of a master console and a surgical manipulator (slave unit) with 2 instrument arms and 1 camera arm. The camera consists of a device with 3-dimensional vision that affords the best possible view of the operative site. The da Vinci surgical endo-wrists, with articulated movements that permit a full 7 degrees of freedom and 360 degrees of rotation, improve maneuverability, making it safe and comfortable to dissect vascular (innominate vein) and nervous (phrenic nerve) structures. All of these characteristics have potentially increased not only the dexterity and safety but also the radicality of conventional video-assisted thoracoscopic thymectomy by enhancing the dissection, particularly for remote and difficult-to-reach areas, of the contralateral mediastinum and neck. A recent study by Rückert and colleagues ${ }^{6}$ retrospectively compared the results of nonrobotic thoracoscopic and robotic thymectomy in patients with MG. A significantly increased rate of CSR (39.2\% vs $20.3 \%$; $P=.01)$ was observed in the robotic group; this result presumably could be related to the better extension and radicality of resection. Most experiences in robotic thymectomy have reported low conversion $(<5 \%)$ and morbidity $(<10 \%)$ rates and short hospital stays ( $<4$ days). ${ }^{7-11}$ Our findings agree with these results, because we had no open conversion, $6 \%$ complications, no nervous or major vascular injuries, and a median hospital stay of 3 days. Our clinical long-term results are in line with recent studies adopting the MGFA recommendations and reporting CSR rates ranging from $28 \%{ }^{17}$ to $42.8 \%{ }^{5}$ for conventional thoracoscopic thymectomy and from $27 \%^{11}$ to $42 \%{ }^{7}$ for robotic thymectomy. Likewise, a wide range of CSR rates have been reported for open transsternal and transcervical approaches $(17 \%-34 \%){ }^{4,18}$ This variability may depend on several factors, including different follow-up durations (18-45 months), heterogeneity of patient characteristics (thymomatous and nonthymomatous, ocular and general MG with different classes of severity, variable length of preoperative symptoms), clinical evaluations not performed by a neurologist, and different practices among neurologists with regard to prescriptions and management strategy for weaning off drugs after thymectomy. Our series suffered from the same limitation bias, because we had a heterogeneous population of patients with both ocular $(11 \%)$ and generalized $\mathrm{MG}$, nonthymomatous and thymomatous $(8 \%) \mathrm{MG}$, and ages less than and more than 40 years $(39 \%)$. It is now fairly well accepted that thymectomy should be offered to young patients with generalized MG. However, uncertainties remain concerning the role of thymectomy for patients with purely ocular symptoms, seronegative disease, late-onset disease, and involvement of ectopic thymic tissue. Between $30 \%$ and $70 \%$ of patients with initial ocular symptoms will eventually develop generalized myasthenia. Thus, some authors ${ }^{19}$ have advocated surgery for patients with purely ocular MG. Our practice is to not exclude these patients, particularly when the symptoms are disabling and poorly controlled by medical therapy. Our results show significantly higher rates of CSR and improvement for those patients with ocular and mildly severe MG. A similar finding was reported by Jaretzki. ${ }^{1}$ In contrast, a review conducted by the American Academy of Neurology reported better clinical improvement after thymectomy in cases with more severe MG. ${ }^{19}$ The indication for thymectomy in AbAchR-negative patients is controversial. This group is heterogeneous; some patients show falsenegative results, whereas others have MuSK antibodies. A retrospective cohort study showed similar postoperative courses in AbAchR-negative and AbAchR-positive patients. ${ }^{20}$ In another study, the presence of MuSK antibodies predicted a poor outcome. ${ }^{21}$ Available evidence suggests that thymectomy should not be recommended in MuSK antibody-positive patients. We followed this recommendation; all seronegative patients were tested for the presence of MuSK antibodies, and only double seronegative patients were eligible for surgery. Our cohort of seronegative patients had a higher, but not significantly higher, CSR rate 
compared with the AbAchR-positive group. In contrast, a significantly poorer improvement rate was observed in seronegative patients. Some studies have found significantly better clinical results in young patients with short disease duration. ${ }^{5,21}$ The robotic procedure has an advantage of being more easily and readily accepted by both neurologists and young patients, because it produces good cosmetic results and is less invasive. In our series, the median duration of preoperative symptoms was 11 months, reflecting an early referral policy and rapid acceptance. Despite no significant difference in CSR based on age and symptom onset, a trend toward CSR was observed in young patients with short $(<1$ year) disease duration before surgery. Rates of detecting ectopic thymic tissue are $22 \%$ to $39.5 \%$ in reported studies and reached $26 \%$ in our experience. Some authors ${ }^{22}$ have highlighted the importance of ectopic thymic tissue in the maintenance of disease and have indicated that it is a negative prognostic factor for remission. Our series of patients with pathologic evidence of ectopic thymus tended to have a lower probability for CSR.

\section{CONCLUSIONS}

Our study reports the outcome of a large group of patients who underwent robotic thymectomy followed by a long follow-up period. We demonstrated that robotic thymectomy is a technically sound operation, with low morbidity and short hospitalization. Neurologic long-term results were good for both CSR and clinical improvement, with a significant reduction in medication over time. In addition, the optimal cosmetic results and minimal invasiveness of this type of surgery were associated with its high acceptance by both patients and neurologists, resulting in early referral and potential further improvement in outcome. A close collaboration between thoracic surgeons and neurologists is important in planning the best treatment strategy to achieve optimal results.

\section{References}

1. Jaretzki A III. Thymectomy for myasthenia gravis: analysis of controversiespatient management. Neurologist. 2003;9:77-92.

2. Masaoka A, Yamakawa Y, Niwa H, Fukai I, Kondo S, Kobayashi M, et al. Extended thymectomy for myasthenia gravis: a 20-year review. Ann Thorac Surg. 1996;62:853-9

3. Jaretzki A, Wolff M. "Maximal" thymectomy for myasthenia gravis. Surgical anatomy and operative technique. J Thorac Cardiovasc Surg. 1988;96:711-6.

4. Shrager JB. Extended transcervical thymectomy: the ultimate minimally invasive approach. Ann Thorac Surg. 2010;89:S2128-34

5. Tomulescu V, Ion V, Kosa A, Sgarbura O, Popescu I. Thoracoscopic thymectomy mid-term results. Ann Thorac Surg. 2006;82:1003-7.

6. Rückert JC, Swierzy M, Ismail M. Comparison of robotic and nonrobotic thoracoscopic thymectomy: a cohort study. J Thorac Cardiovasc Surg. 2011;141: 673-7.

7. Ruckert JC, Ismail M, Swierzy M, Sobel H, Rogalla P, Meisel A, et al. Thoracoscopic thymectomy with the da Vinci robotic system for myasthenia gravis. Ann N Y Acad Sci. 2008;1132:329-35.

8. Rea F, Marulli G, Bortolotti L, Feltracco P, Zuin A, Sartori F. Experience with the "da Vinci" robotic system for thymectomy in patients with myasthenia gravis: report of 33 cases. Ann Thorac Surg. 2006;81:455-9.
9. Cakar F, Werner P, Augustin F, Schmid T, Wolf-Magele A, Sieb M, et al. A comparison of outcomes after robotic open extended thymectomy for myasthenia gravis. Eur J Cardiothorac Surg. 2007;31:501-5.

10. Goldstein SD, Yang SC. Assessment of robotic thymectomy using the Myasthenia Gravis Foundation of America Guidelines. Ann Thorac Surg. 2010;89: 1080-6.

11. Freeman RK, Ascioti AJ, Van Woerkom JM, Vyverberg A, Robison RJ. Long term follow-up after robotic thymectomy for nonthymomatous myasthenia gravis. Ann Thorac Surg. 2011;92:1018-23.

12. Jaretzki A, Barohn RJ, Ernstoff RM, Kaminski HJ, Keesey JC, Penn AS, et al Myasthenia gravis: recommendations for clinical research standards. Ann Thorac Surg. 2000;70:327-34.

13. Rückert JC, Czyzewski D, Pest S, Müller JM. Radicality of thoracoscopic thymectomy-an anatomical study. Eur J Cardiothorac Surg. 2000;18:735-6.

14. Newsom-Davis J, Cutter G, Wolfe GI, Kaminski HJ, Jaretzki A III, Minisman G et al. Status of the thymectomy trial for nonthymomatous myasthenia gravis patients receiving prednisone. Ann N Y Acad Sci. 2008;1132:344-7.

15. Ruckert JC, Walter M, Muller JM. Pulmonary function after thoracoscopic thymectomy versus median sternotomy for myasthenia gravis. Ann Thorac Surg. 2000;70:1656-61.

16. Savitt MA, Gao G, Furnary AP, Swanson J, Gately HL, Handy JR. Application of robotic-assisted techniques to the surgical evaluation and treatment of the anterior mediastinum. Ann Thorac Surg. 2005;79:450-5.

17. Keating CP, Kong YX, Tay V, Knight SR, Clarke CP, Wright GM. VATS thymectomy for nonthymomatous myasthenia gravis: standardized outcome assessment using the myasthenia gravis foundation of America clinical classification. Innovations. 2011;6:104-9.

18. Kattach H, Anastasiadis K, Cleuziou J, Buckley C, Shine B, Pillai R, et al. Transsternal thymectomy for myasthenia gravis: surgical outcome. Ann Thorac Surg. 2006;81:305-8.

19. Gronseth GS, Barohn RJ. Practice parameter: thymectomy for autoimmune myasthenia gravis (an evidence-based review): report of the Quality Standards Subcommittee of the American Academy of Neurology. Neurology. 2000;55: 7-15.

20. Guillermo GR, Tellez-Zenteno JF, Weder-Cisneros N, Mimenza A, Estañol B Remes-Troche JM, et al. Response of thymectomy: clinical and pathological characteristics among seronegative and seropositive myasthenia gravis patients. Acta Neurol Scand. 2004;109:217-21.

21. Pompeo E, Tacconi F, Massa R, Mineo D, Nahmias S, Mineo TC. Long-term outcome of thoracoscopic extended thymectomy for nonthymomatous myasthenia gravis. Eur J Cardiothorac Surg. 2009;36:164-9.

22. Ponseti JM, Gamez J, Vilallonga R, Ruiz C, Azem J, López-Cano M, et al. Influence of ectopic thymic tissue on clinical outcome following extended thymectomy in generalized seropositive nonthymomatous myasthenia gravis. Eur $J$ Cardiothorac Surg. 2008;34:1062-7.

\section{Discussion}

Dr Mithran Sukumar (Portland, Ore). You were succinct in presenting your surgical results and neurologic outcomes after thymectomy for myasthenia, particularly in the effect that it has on the postoperative management of these patients. I have 2 questions for you.

The first is in regard to bleeding with robotic thymectomy. Would you briefly discuss the possible sites and causes? Is there an incidence of catastrophic bleeding, and how would you manage that?

Dr Marulli. All patients with postoperative bleeding did not have a re-do operation, so we can suppose looking at the chest $\mathrm{x}$-ray that the bleeding was from one of the ports, probably in 2 cases from the port of the camera and in 2 cases from the port of the parasternal area because these ports receive stress during maneuvers and the parasternal port is the port that we use to remove the specimen; thus, it may be damaged. There is no relationship with the experience. Recently, we had another case of bleeding in which we performed reoperation. The bleeding was from the thoracic vessels, probably because the resident damaged the 
vessels during the small opening of the access. There was no intraoperative bleeding, but eventually its management could be difficult, probably requiring an open conversion.

Dr Sukumar. My second question is to deal with this whole idea of robotic thymectomy. Your slide presented the possible advantages of robotic thymectomy, but would you discuss the real disadvantages at this time?

Dr Marulli. There are some disadvantages. Some are important and some are less important. For example, one of the most common disadvantages is the absence of tactile feedback. This is not an important disadvantage because superior vision is enough to overcome this limitation. The main technical disadvantage is probably when you have bleeding from a large vessel. The necessity for emergency conversion can be more difficult for 2 reasons. The first reason is that the main surgeon is far from the patient and is not scrubbed, so you should have an assistant who should be able to do an emergency conversion. Moreover, the emergency conversion can be difficult because the robot is over the patient, so you do not have much space. On the other hand, the control of the vessel may be better because of the high degree and complexity of the movements allowed by robotic instruments, so you can grasp the vessel to temporarily control the bleeding. Another limitation is the cost. The fixed cost can be reduced by using the robot in a multidisciplinary setting. In our hospital we use the robot with the urologist, gynecologist, and general surgeons. Although the cost of the disposable materials is obviously high, it is not recoverable. Another limitation is the absence of suction devices, which can be a limitation not only for the thymectomy, which is an easy technique with no need for suction, but also for the lobectomy.

Dr Joseph Shrager (Stanford, Calif). All the robotic talking points that we commonly hear about are nice (eg, reduction of tremor), and it is probably fun to do a thymectomy that way. But we have published that transcervical thymectomy has almost no cost, there is no fancy equipment, and we have a higher CSR rate than you have published. You can also do it as an outpatient procedure. There is essentially no pain for the patient and almost no morbidity. The only bad thing about that procedure is that it is hard to find places where people can learn how to do it. It is hard to justify all the costs of robot-given the talks we have heard during this whole meeting about economics—when you can do this thymectomy quickly and with at least as good results with nothing other than a little substernal retractor.

Dr Marulli. I agree with you. I know your work, and I think transcervical thymectomy can become the standard of care in this kind of patient. We need more reliable results because at the time only a few articles address these types of results. Moreover, I have 2 concerns. Although I never used the transcervical approach, my chief had a good experience with this approach, and he considers the transcervical technique somewhat difficult to learn in comparison with the robot technique, so my concern is the learning curve. Moreover, your experience is the best experience. You have $8 \%$ open conversion if I remember, probably split conversion, which is higher than the robotic technique. We have $0 \%$ conversion, so I do not know if the technique is ready to be widely used, but it absolutely is the best of mini-invasive techniques in my opinion.

Dr Shrager. Almost all the conversions were in cases that I would not now recommend the procedure be attempted, where we were trying to do it for a small thymoma, and I would not recommend it for those cases. Also, you learn after a while that it cannot be done if the patient cannot really extend his or her neck well. It is not hard to learn; it is just that there are only a few places or people who can teach you how to do it. I mean, the residents can do it after basically seeing one of them. It is simple, and I would invite anybody who wants to learn how to do it. Cases are not all that frequent, but anyone who wants to learn could come to Stanford and see how it is done.

Dr Marulli. I like the transcervical thymectomy. My experience in the robotic thymectomy was positive, and the learning curve with robotic technique was easy.

Dr David Follette (Sacramento, Calif). I agree with Joe about the transcervical thymectomy. Over the years at this meeting, we have heard a number of talks. I have never seen a nicer one describing the robotic technique, but my comment and question is not can you do it but should you do it? The whole field of robotic surgery with few exceptions has never been proven to improve long-term outcomes or mortality. It improves cosmetics; you do not get a keloid. As we learned this morning, procedures that are fancy and look great on the Internet may not really be justified unless there are outcomes. Thoracic surgery has spent a lot of our time, especially in the world of oncology, trying to figure out what is best for the patient before adopting new techniques. My concern is, and my philosophy has evolved, if you have 2 techniques and your patient is worried about cosmetics and wants to pay the extra $\$ 35,000$ out of pocket, so be it unless you really have evidence that the long-term outcome, not just 1 day shorter in the hospital or nicer scar, is better. Have you compared, because you have an extensive experience and a wonderful team, the actual long-term outcome of standard techniques versus robotic techniques in terms of certain patients who have recidivism of their myasthenia symptoms long-term and increased doses of medication. Have you made an effort to look at the long-term outcomes, and can you honestly say there is a distinct benefit to the patient that justifies the significant increase in cost other than cosmesis? People in the United States, if they want cosmetic surgery, they pay for it out of their pockets.

Dr Marulli. I do not have a reply for this question because we did not compare with the previous technique, and a retrospective comparison is not useful, so we have to wait for major data from studies adopting the MGFA classification, the same criteria. We shifted from the classic conventional video-assisted thoracoscopic approach to the robotic approach. From a technical point of view, there is a big difference. In our previous experience, we had at least $30 \%$ of patients who required a further transcervical approach and approximately $10 \%$ of patients required an open conversion, so from a technical point of view, the resection is safer, bigger, and more radical. For long-term results, I have no reply for you. 